\title{
FAST EXTRACTION KICKER FOR THE ACCELERATOR TEST FACILITY*
}

\author{
S. De Santis", LBNL, Berkeley, California \\ J. Urakawa, T. Naito, KEK, Ibaraki, Japan.
}

\begin{abstract}
We present the results of a study for the design of a fast extraction kicker to be installed in the Accelerator Test Facility ring at KEK. This activity is carried on in the framework of the ATF2 project, which will be built on the KEK Tsukuba campus as an extension of the existing ATF, taking advantage of the world's smallest normalized emittance achieved there. ATF2's primary goal is to operate as a test facility and establish the hardware and beam handling technologies envisaged for the International Linear Collider. In particular, the fast extraction kicker object of the present paper is an important component of the ILC damping rings, since its rise and fall time define the minimum distance between bunches and ultimately the damping rings length itself. Building on the initial results presented at EPAC '06, we report on the present status of the kicker design and define the minimum characteristics for pulsers and other subsystems. In addition to the original scheme with multiple stripline modules producing a total deflection of $5 \mathrm{mrad}$, we also investigated a scheme with a single kicker module for a reduced deflection of $1 \mathrm{mrad}$ placed inside a closed orbit bump, which takes the electron closer to the extraction septum.
\end{abstract}

\section{INTRODUCTION}

We have already calculated the fundamental parameters of a kicker for single bunch extraction in the ATF [1]. Given the space limitation in the ring, we chose to operate with three stripline modules placed in two different locations, each $65 \mathrm{~cm}$ long. To minimize the kicker's impact on the beam impedance we chose to have a $24 \mathrm{~mm}$ distance between electrodes as our target. This distance is equal to the beam pipe diameter, so that discontinuities from the bunch wake field point of view are minimized as possible. The coverage angle was chosen equal to 120 for each stripline electrode. This is a classic configuration for stripline kickers, since smaller angles reduce the kicking field, while larger angles increase the beam coupling impedance and require larger beam pipes to obtain a $50 \Omega$ characteristic impedance, although they don't result in a substantial reduction of the required voltage.

In Tab. 1 we summarize the calculated kicker parameters for the chosen configuration, together with the main specifications of the project.

In the following section we show our computer simulation using the EM modeling suite Microwave Studio [2], where we studied the electromagnetic fields time evolution in correspondence of a complete cycle, from the beginning of a pulse to the passage of the next trailing bunch through a kicker's module.

We also studied the uniformity of the kicking field and calculated the kicker's S-parameters, its coupling and transfer impedance.

In the third section, we then repeat the calculations for a different kicker configuration utilizing a single $50 \mathrm{~cm}$ long module for a $1 \mathrm{mrad}$ deflection inside a closed orbit bump.

Finally, we discuss some critical aspects concerning the kicker's pulsers and feedthroughs.

Table 1: Relevant ATF parameters and fast kicker specifications (3-modules configuration).

\begin{tabular}{|l|l|}
\hline Beam energy $\mathbf{E}_{\mathbf{b}}$ & $1.28 \mathrm{GeV}$ \\
\hline Beam pipe radius $\mathbf{r}_{\mathbf{p}}$ & $12 \mathrm{~mm}$ \\
\hline Bunch spacing $\mathbf{t}_{\mathbf{b}}$ & $5.6 \mathrm{~ns}$ \\
\hline Deflection angle $\boldsymbol{\theta}_{\mathbf{k}}$ & $5 \mathrm{mrad}$ \\
\hline Kick repetition rate $\mathbf{f}_{\text {rep }}$ & $3 \mathrm{MHz}$ \\
\hline Total kicker length $\mathbf{L}_{\mathbf{T}}$ & $1.4+0.8 \mathrm{~m}$ \\
\hline Kicker field at $\mathrm{t}=\mathrm{t}_{0}+\mathrm{t}_{\mathrm{b}} \mathbf{E}\left(\mathbf{t}_{\mathbf{0}}+\mathbf{t}_{\mathbf{b}}\right)$ & $<7 \cdot 10^{-4} \mathrm{E}\left(\mathrm{t}_{0}\right)$ \\
\hline Required stripline vgoltage $\mathbf{V}_{\mathbf{k}} / \mathbf{2}$ & $21 \mathrm{kV}$ \\
\hline Shunt impedance $\mathbf{R}_{\mathbf{s}}$ & $250 \mathrm{k} \Omega$ \\
\hline Peak power $\mathbf{P}_{\mathbf{k}}$ & $7 \mathrm{~kW}$ \\
\hline Average power $($ at $3 \mathrm{MHz}) \mathbf{P}_{\text {avg }}$ & $100 \mathrm{~W}$ \\
\hline
\end{tabular}

\section{COMPUTER 3D ELECTROMAGNETIC MODELLING OF THE KICKER}

Following the analytical calculation and 2D electromagnetic modeling, we undertook a careful threedimensional computer model of on of our kicker module to described in [2]. Figure 1 shows a view of our Microwave Studio 3D model of a $650 \mathrm{~mm}$ long stripline module with 5:1 tapers. We designed some simple $50 \Omega$ feedthroughs, with Teflon dielectric and standard N-Type connector dimensions. It will be discussed in a later section how, in fact, the choice of an appropriate highpower, high-voltage feedthrough is one of the critical points in the kicker design. We used this simple connector in order to be able to proceed in the analysis described in the following. Once a suitable feedthrough is identified the striplines will be optimized to ensure good impedance matching. Also, in Fig.1 one cans see the 5:1 ratio tapers, which are used to reduce the kicker impedance,

\footnotetext{
*Work supported by the U.S. Department of Energy under Contract No. DE-AC0-05CH11231.

"sdesantis@1bl.gov
} 
connecting the $22 \mathrm{~mm}$ radius kicker chamber to the 12 $\mathrm{mm}$ beam pipe.

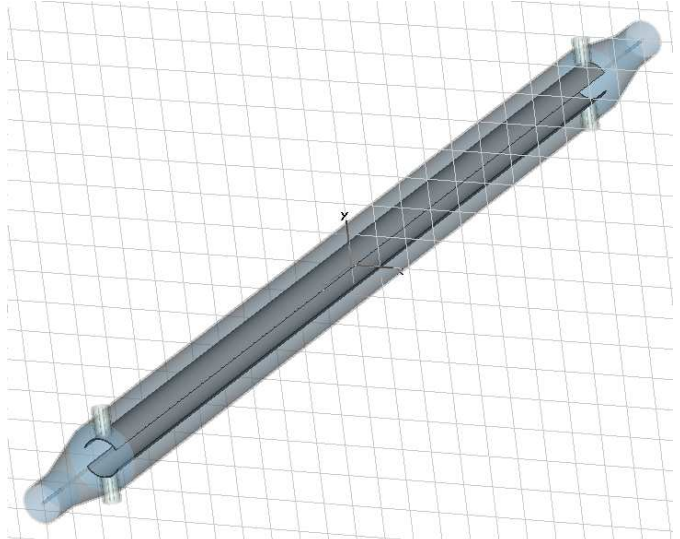

Fig.1. View of the kicker CAD model (Microwave Studio) with tapers, feedthroughs and coaxial wire.

Finally, Fig. 1 also shows a coaxial wire of $2 \mathrm{~mm}$ diameter running through the module. This is of course no part of the kicker, but we used it to measure coupling and transfer impedance, by realizing a "virtual" measurement of the S-parameters, the same way as this is done in "hardware" with the classic coaxial-wire method for bench measurements.

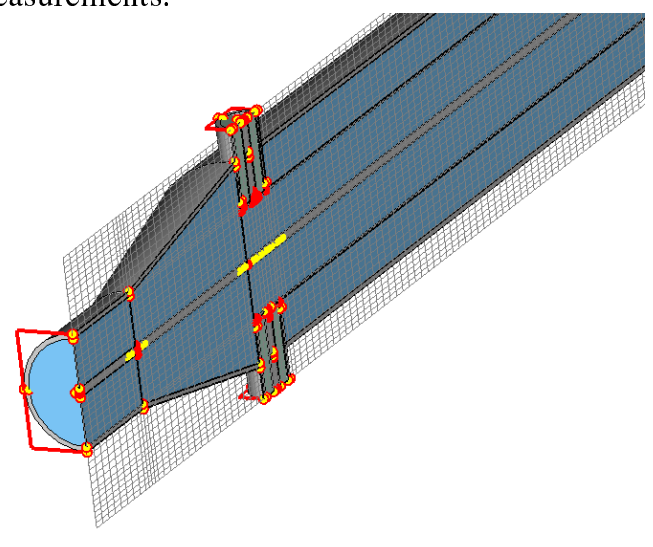

Meshplane at $x=\quad \theta($ Index $=18$ )

Fig.2. Detail of the simulation mesh in correspondence of one of the kicker extremities.

Figure 2 shows the automatically generated mesh on the module mid-section. We choose a minimum of 15 meshpoints per wavelength, which are increased in correspondence of critical points.

Microwave Studio is an FDTD based code and therefore especially well suited for analyzing the transient behaviour of the kicker in correspondence of a given excitation waveform. We used an excitation voltage with a $\mathrm{t}_{\mathrm{ft}}=5.45 \mathrm{~ns}$ long flat top which allows for an extra $1 \mathrm{~ns}$ filling time, compared to the theoretical $4.34 \mathrm{~ns}$ ( 2 times kicker length divided by the speed of light), in order to reach a more uniform deflecting field. Rise time $t_{r}=150 \mathrm{ps}$ and fall time $t_{d}=300$ ps were chosen according to the current state of the art for fast pulsers [3]. The simulation was extended to $11.3 \mathrm{~ns}$, corresponding to the passage through the module of the trailing bunch.

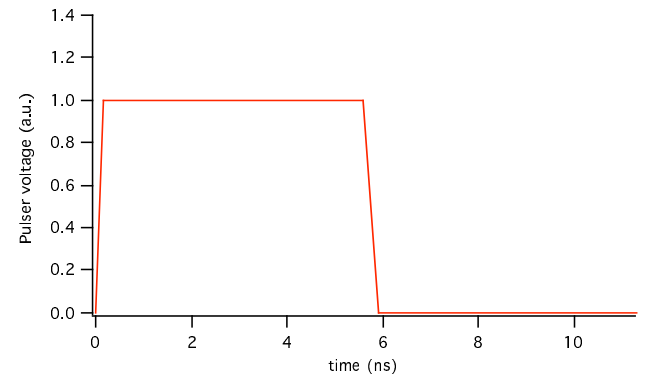

Fig.3. Pulser waveform.

The pulser waveform is shown in Fig.3.

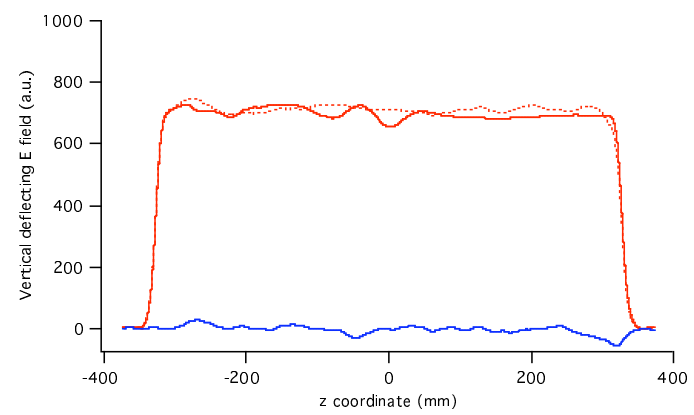

Fig.4. Vertical electric field $\left(\mathrm{E}_{\mathrm{y}}\right)$ along the nominal orbit when the bunch enters module (solid red); bunch is at module mid-length (dashed red); trailing bunch enters module (blue).

Figure 4 shows the vertical deflecting field Ey calculated along the bunch ideal orbit. By comparing the solid red curve to the dashed one, taken with the bunch at $\mathrm{z}=-350$ $(1 \mathrm{~ns}$ after the pulser firing) and $\mathrm{z}=0 \mathrm{~mm}(2.1 \mathrm{~ns}$ after pulser firing) respectively, we can evaluate how $1 \mathrm{~ns}$ prefiring of the pulser is sufficient to have the deflecting field reach a reasonably good flatness already when the bunch enters the module. The blue curve shows how much the field value has decayed when the trailing bunch reaches the module's mid-length, its average value being reduced to $5 \cdot 10^{-3}$ times.

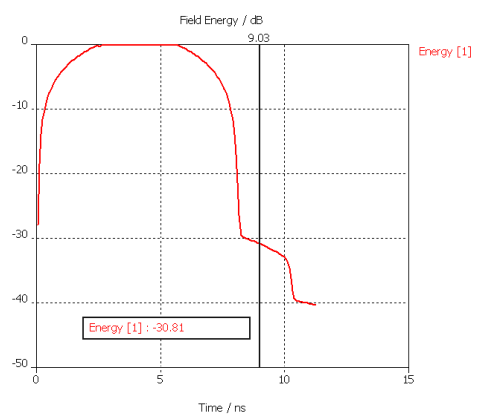

Fig.5. Normalized energy stored in a stripline module.

Residual field levels can also be evaluated by looking at the time evolution of the energy stored in the module (Fig.5), which we calculated to be at least $30 \mathrm{~dB}$ below it's peak value. In addition, one has to consider that this is a free oscillating field and the residual level in a real kicker will be lower due to ohmic losses. 


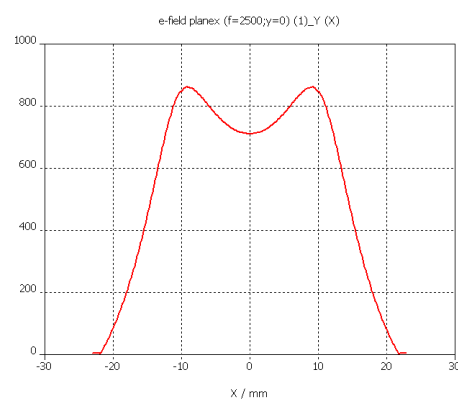

Fig.6. Transverse distribution of deflecting field at kicker's mid-length.

Figure 6 shows the transverse distribution of the kicking field calculated in correspondence of the kicker midlength. The field variation is below $1 \%$ of its central value over an excursion of $5 \mathrm{~mm}$ across the bunch nominal orbit.

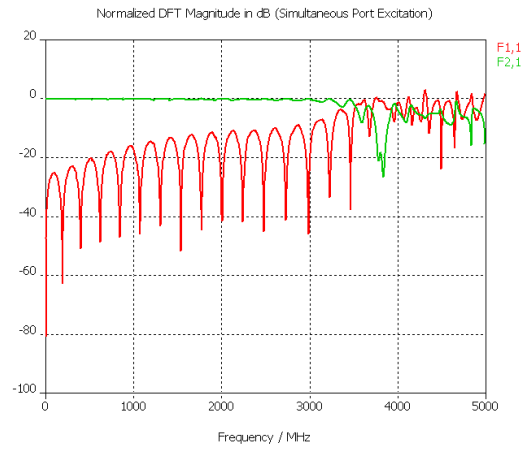

Fig.7. Calculated S-parameters between upstream and downstream feedthroughs: reflection (red) and transmission (green).

In Fig. 7 we show the calculated S-parameters between the two stripline ports, to verify impedance matching of the feedthroughs to the striplines. Even with our simple design there is an acceptable matching below $3 \mathrm{GHz}$, which will be improved after choosing the actual feedthroughs.

\section{Coaxial wire simulations}

We also calculated the kicker longitudinal impedance simulating the classic coaxial wire method using Walling's formula for distributed impedances [4]

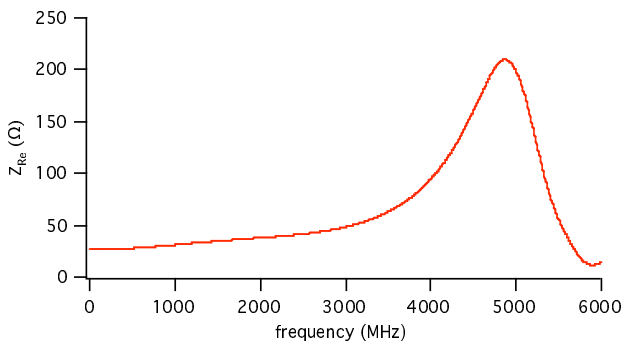

Fig.8. Real part of the longitudinal impedance.

With the data in Fig. 8 it is possible to calculate the power deposition on the striplines and the beam energy loss (loss factor). We analogously calculated the transfer impedance on both up and downstream ports.

\section{SINGLE KICKER MODULE WITH CLOSED ORBIT BUMP}

We substantially repeated the calculations shown in [1] for a different extraction configuration where only $1 \mathrm{mrad}$ deflection is required, but the kicker length has to be less than $60 \mathrm{~cm}$. Table 2 shows those kicker's parameters, which are different from the original configuration.

Table 2: Relevant ATF parameters and fast kicker specifications (3-modules configuration).

\begin{tabular}{|l|l|}
\hline Deflection angle $\boldsymbol{\theta}_{\mathrm{k}}$ & $1 \mathrm{mrad}$ \\
\hline Total kicker length $\mathbf{L}_{\mathbf{T}}$ & $0.6 \mathrm{~m}$ \\
\hline Required stripline vgoltage $\mathbf{V}_{\mathbf{k}} / \mathbf{2}$ & $16.5 \mathrm{kV}$ \\
\hline Shunt impedance $\mathbf{R}_{\text {s }}$ & $150 \mathrm{k} \Omega$ \\
\hline Peak power $\mathbf{P}_{\mathbf{k}}$ & $1.8 \mathrm{~kW}$ \\
\hline Average power (at $3 \mathrm{MHz}$ ) $\mathbf{P}_{\text {avg }}$ & $30 \mathrm{~W}$ \\
\hline
\end{tabular}

One can notice how, due to the lower average power it would be possible in principle to use in-vacuum ferrite loads in case a shorter field decay time is desired.

We run a $3 \mathrm{D}$ computer simulation for the transient response for this kicker as well and calculated its impedance. Our results don't suggest any additional problems.

\section{CONCLUSIONS}

From the results presented in this paper we can recognize the two critical aspects in the realization of a fast extraction kicker, namely: pulser time response and maximum repetition rate, and feedthroughs power handling capabilities. Both aspects are strictly correlated to the $3 \mathrm{MHz}$ repetition rate chosen, since pulsers with even higher voltage than needed and sufficient wavefronts steepness are already available on the market for lower repetition rates. Also, the feedthroughs, which in our case have to withstand powers above $100 \mathrm{~kW}$, could be bought off the shelf. Nonetheless, the design of a 3-6 MHz $10 \mathrm{kV}$ pulser is underway and we have identified a possible solution for the feedthrough, which we are planning to test in early 2008.

\section{REFERENCES}

[1] S. De Santis, A Wolski and M. Ross "Design of a Fast Extraction Kicker for the Accelerator Test Facility", in Proc. of EPAC 2006, Edinburgh (2006)

[2] http://www.cst.com

[3] FID GmbH. http;//www.fidtechnology.com

[4] L.S. Walling, et al. Nucl. Instrum. Methods Phys. Res. A 281, 433 (1989). 